\title{
SCALING SETS AND ORTHONORMAL WAVELETS WITH DILATIONS INDUCED BY EXPANDING MATRICES
}

\author{
DAMIR BAKIĆ AND EDWARD N. WILSON \\ University of Zagreb, Croatia and Washington University in St. Louis, USA
}

\begin{abstract}
The paper studies orthonormal wavelets in $L^{2}\left(\mathbb{R}^{n}\right)$ with dilations induced by expanding integer matrices of arbitrary determinant. We provide a method for construction of all scaling sets and, hence, of all orthonormal MSF wavelets with the additional property that the core space of the underlying multiresolution structure is singly generated. Several examples on the real line and in $\mathbb{R}^{2}$ are included. We also prove that all MSF orthonormal wavelets whose dimension function is essentially bounded by 1 are obtained by our construction method. Finally, we derive a description of all wavelets (not necessarily MSF ones) that arise from a single scaling function in terms of the underlying multiresolution structure.
\end{abstract}

\section{INTRODUCTION}

The classical Shannon wavelet with dyadic dilations on the real line is given by $\hat{\psi}=\chi_{\left[-1,-\frac{1}{2}\right) \cup\left[\frac{1}{2}, 1\right)}=\chi_{2 I \backslash I}$ where $I$ denotes the unit interval $\left[-\frac{1}{2}, \frac{1}{2}\right)$ and $\hat{\psi}$ is the Fourier transform of $\psi$. This is the simplest representative of the class of MSF MRA orthonormal wavelets. By MSF ("minimally supported frequency") one refers to the fact that there is a set $W$ called a wavelet set such that $\hat{\psi}$ is of the form $\chi_{W}$. Another important feature of the Shannon wavelet is the fact that it arises from a multiresolution analysis (MRA).

Recall that a multiresolution analysis (MRA) in $L^{2}(\mathbb{R})$ is a sequence $\left(V_{j}\right), j \in \mathbb{Z}$, of closed subspaces of $L^{2}(\mathbb{R})$ with the properties

(i) $V_{j} \subseteq V_{j+1}, \forall j$;

(ii) $D V_{j}=V_{j+1}, \forall j$;

(iii) $\cap_{j \in \mathbb{Z}} V_{j}=\{0\}, \overline{\cup_{j \in \mathbb{Z}} V_{j}}=L^{2}(\mathbb{R})$;

2010 Mathematics Subject Classification. 42A99, 42C15.

Key words and phrases. Expanding matrix, orthonormal wavelet, scaling set, multiresolution analysis. 
(iv) there exists $\varphi \in V_{0}$ such that the system $\left\{T_{k} \varphi: k \in \mathbb{Z}\right\}$ is an orthonormal basis for $V_{0}$.

Here $D$ and $T_{k}, k \in \mathbb{Z}$, denote dilation and translation operators, respectively: $D f(x)=\sqrt{2} f(2 x), T_{k} f(x)=f(x-k)$. The function $\varphi$ from (iv) is called a scaling function, and condition (iv) is usually described by saying that $\varphi$ is an orthonormal generator for the core space $V_{0}$.

Given an MRA $\left(V_{j}\right)$, we define, for all $j \in \mathbb{Z}, W_{j}=V_{j+1} \ominus V_{j}$. One then easily obtains $D W_{j}=W_{j+1}, \forall j$, and $L^{2}(\mathbb{R})=\oplus_{j \in \mathbb{Z}} W_{j}$. Moreover, it turns out that there is a function $\psi \in W_{0}$ such that the system $\left\{T_{k} \psi: k \in \mathbb{Z}\right\}$ is an orthonormal basis for $W_{0}$ and, consequently, $\psi$ is an orthonormal wavelet. Each orthonormal wavelet obtained in this way is called an MRA wavelet. The MRA concept is well known; for a comprehensive discussion of MRA wavelets we refer to Chapters 2 and 7 in [13]. In the case of the Shannon wavelet the underlying scaling function $\varphi$ is given by $\hat{\varphi}=\chi_{I}$; in this situation, when the Fourier transform of a scaling function $\varphi$ of an MRA is of the form $\hat{\varphi}=\chi_{S}$, we say that $S$ is a scaling set.

A characterization of scaling sets is known; in addition, there is a simple relation between scaling sets and the corresponding wavelet sets.

Before stating the relevant result, let us recall the definition of a $\mathbb{Z}$-tiling domain: a measurable set $F \subseteq \mathbb{R}$ is said to be a $\mathbb{Z}$-tiling domain if, for a.e. $\xi \in \mathbb{R}$, there exists a unique $k \in \mathbb{Z}$ such that $\xi+k \in F$.

The following proposition (easily deduced from [13, Theorem 7.5.2 and Proposition 2.2.13], see also [15, Theorem 8]) is known.

Proposition 1.1. A measurable set $S \subseteq \mathbb{R}$ is a scaling set if and only if the following conditions are satisfied:

(i) $S$ is a $\mathbb{Z}$-tiling domain;

(ii) $\frac{1}{2} \xi \in S$ for a.e. $\xi \in S$;

(iii) for a.e. $\xi \in \mathbb{R}$, there exists $j_{0} \in \mathbb{N}$ such that $\frac{1}{2^{j}} \xi \in S, \forall j \geq j_{0}$.

If $S$ is a scaling set, the function $\psi$ defined by $\hat{\psi}=\chi_{2 S \backslash S}$ is an orthonormal wavelet. Conversely, each MSF MRA orthonormal wavelet $\psi$ is of the form $\hat{\psi}=\chi_{2 S \backslash S}$ where $S$ is a scaling set.

The aim of the present article is to generalize this proposition to the case of dilations in $L^{2}\left(\mathbb{R}^{n}\right)$ induced by expanding matrices with integer coefficients of arbitrary determinant. We shall find a suitable analog of the concept of a scaling set for such dilations and provide both a characterization and a construction method of scaling sets.

In the remaining part of this introductory section we summarize all the relevant results known from the literature. This will also explain the difficulty that arises when dealing with general dilations and a very special role played by the dilation factor 2 . 
Let us fix necessary notations. Throughout the paper $A \in M_{n}(\mathbb{Z})$ will denote an arbitrary expanding matrix (by expanding, we mean that all eigenvalues of $A$ have absolute value greater than 1). We denote by $B$ the transpose of $A$. Let $d=|\operatorname{det} A|=|\operatorname{det} B|$; clearly, $d \in \mathbb{N}$, and $d \geq 2$. Recall that $\mathbb{Z}^{n} / B \mathbb{Z}^{n}$ and $B^{-1} \mathbb{Z}^{n} / \mathbb{Z}^{n}$ are groups of order $d$. In the sequel we shall fix a complete set of representatives $\left\{\alpha_{0}=0, \alpha_{1}, \ldots, \alpha_{d-1}\right\}$ for the quotient group $\mathbb{Z}^{n} / B \mathbb{Z}^{n}$. Note that the set $\left\{\beta_{0}=0, \beta_{1}, \ldots, \beta_{d-1}\right\}$, with $\beta_{j}=B^{-1} \alpha_{j}$, is then a complete set of representatives for $B^{-1} \mathbb{Z}^{n} / \mathbb{Z}^{n}$.

Let $D$ and $T_{k}, k \in \mathbb{Z}^{n}$, denote our dilation and translation operators on $L^{2}\left(\mathbb{R}^{n}\right)$, respectively: $D f(x)=\sqrt{d} f(A x), T_{k} f(x)=f(x-k)$.

A function $\psi \in L^{2}\left(\mathbb{R}^{n}\right)$ is an orthonormal wavelet (more precisely, we shall say $A$-wavelet in order to specify the underlying dilation matrix $A$ when needed) if the system $\left\{D^{j} T_{k} \psi: j \in \mathbb{Z}, k \in \mathbb{Z}^{n}\right\}$ is an orthonormal basis for $L^{2}\left(\mathbb{R}^{n}\right)$.

To each orthonormal wavelet $\psi$ one can attach a so called dimension function $D_{\psi}$ defined by $D_{\psi}(\xi)=\sum_{j=1}^{\infty} \sum_{k \in \mathbb{Z}^{n}}\left|\hat{\psi}\left(B^{j}(\xi+k)\right)\right|^{2}$. It is well known that $D_{\psi}$ is well defined a.e. and $\mathbb{Z}^{n}$-periodic. Moreover, we have $\int_{\mathbb{T}} D_{\psi}(\xi) d \xi=1$ where $\mathbb{T}$ denotes the $n$-dimensional torus. For these and other facts concerned with dimension functions we refer the reader to [7].

Let us now turn to the concept of a generalized multiresolution analysis which is another useful tool in our study.

Definition 1.2. A sequence $\left(V_{j}\right)$ of closed subspaces of $L^{2}\left(\mathbb{R}^{n}\right)$ is called a generalized $A$-multiresolution analysis (A-GMRA, or simply GMRA) if the following conditions are satisfied:

(i) $V_{j} \subseteq V_{j+1}, \forall j$;

(ii) $D V_{j}=V_{j+1}, \forall j$;

(iii) $\cap_{j \in \mathbb{Z}} V_{j}=\{0\}, \overline{\cup_{j \in \mathbb{Z}} V_{j}}=L^{2}\left(\mathbb{R}^{n}\right)$;

(iv) $V_{0}$ is a shift invariant space, i.e. $f \in V_{0} \Rightarrow T_{k} f \in V_{0}, \forall k \in \mathbb{Z}^{n}$.

For a GMRA $\left(V_{j}\right)$ we define, as in the multiresolution case, $W_{j}=V_{j+1} \ominus$ $V_{j}, j \in \mathbb{Z}$. Again, it turns out that $D W_{j}=W_{j+1}, \forall j$, and $L^{2}\left(\mathbb{R}^{n}\right)=\oplus_{j \in \mathbb{Z}} W_{j}$. Thus, if we can find a function $\psi \in W_{0}$ such that the system $\left\{T_{k} \psi: k \in \mathbb{Z}^{n}\right\}$ is an orthonormal basis for $W_{0}$, it follows immediately that $\psi$ is an orthonormal wavelet. When this is the case, we say that $\left(V_{j}\right)$ admits orthonormal wavelets and each orthonormal wavelet that arises in the way described above is said to be associated with $\left(V_{j}\right)$.

The importance of the concept of a GMRA lies in the fact that each orthonormal wavelet $\psi$ is associated with a GMRA: one simply takes $V_{0}$ as the closed linear span of the set $\left\{D^{j} T_{k} \psi: j<0, k \in \mathbb{Z}^{n}\right\}$ and $V_{j}=D^{j} V_{0}, j \in \mathbb{Z}$. In the dyadic case on the real line this is first observed in [14].

However, there is an important difference between GMRA's and MRA's. If $\left(V_{j}\right)$ is an MRA with dyadic dilations on the real line, then $W_{0}$ always contains a function $\psi$ such that $\left\{T_{k} \psi: k \in \mathbb{Z}\right\}$ is an orthonormal basis for 
$W_{0}$; in other words, $\left(V_{j}\right)$ admits orthonormal wavelets. In contrast, GMRA's need not allow orthonormal wavelets. To explain the difficulty, we need a theorem from [5] that describes the structure of shift invariant spaces. First, recall that a sequence $\left(x_{n}\right)$ in a separable Hilbert space $H$ is a Parseval frame for $H$ if $\|x\|^{2}=\sum_{n=1}^{\infty}\left|\left\langle x, x_{n}\right\rangle\right|^{2}, \forall x \in H$.

REMARK 1.3. (a) ([5, Theorem 3.3.]) Let $V \subseteq L^{2}\left(\mathbb{R}^{n}\right)$ be a shift invariant space. Then there is a sequence (possibly finite) $\left(V_{j}\right)$ of subspaces of $V$ such that $V=\oplus_{j=1}^{\infty} V_{j}$ and, for each $j$, there exists a function $\varphi_{j} \in V_{j}$ such that the system $\left\{T_{k} \varphi_{j}: k \in \mathbb{Z}^{n}\right\}$ is a Parseval frame for $V_{j}$ (in this situation we say that $\varphi_{j}$ is a Parseval generator for $V_{j}$ and write $\left.V_{j}=\left\langle\varphi_{j}\right\rangle\right)$.

(b) It is well known that, in the above situation, for each $j$ there exists a measurable $\mathbb{Z}^{n}$-periodic set $\Omega_{j}$ with the property $\sum_{k \in \mathbb{Z}^{n}}\left|\hat{\varphi}_{j}(\xi+k)\right|^{2}=\chi_{\Omega_{j}}(\xi)$ a.e. In addition, the system $\left\{T_{k} \varphi_{j}: k \in \mathbb{Z}^{n}\right\}$ is an orthonormal basis for $V_{j}$ if and only if $\Omega_{j}=\mathbb{R}^{n}$, up to a set of measure zero. The subspaces $\left(V_{j}\right)$ in the above decomposition can be arranged in such a way that $\Omega_{1} \supseteq \Omega_{2} \supseteq$...

(c) The functions $\varphi_{j}$ from (a) are not uniquely determined by $V$. However, the dimension function $\operatorname{dim}_{V}$ of $V$ (see [5] for the details) satisfies $\operatorname{dim}_{V}(\xi)=$ $\sum_{j=1}^{\infty} \chi_{\Omega_{j}}(\xi)$ a.e.; thus, the sets $\Omega_{j}$ are determined by $V$, up to null-sets, in a unique way.

In view of the above remark we see that a GMRA $\left(V_{j}\right)$ is an MRA precisely when the core space $V_{0}$ is singly generated as a shift invariant space and, additionally, when $V_{0}$ possesses an orthonormal generator. Singly generated core spaces will play the central role in our study; however, it will be seen that it is necessary for our purposes to work with those singly generated core spaces that admit only Parseval (and not orthonormal) generators. Such GMRA's can be called Parseval frame multiresolution analyses (PF MRA's).

PF MRA's were first studied in [4] for dyadic dilations on the real line. We also refer the reader to [3] for an extensive study of PF MRA's in $L^{2}\left(\mathbb{R}^{n}\right)$ with dilations induced by expanding matrices $A$ with the property $|\operatorname{det} A|=2$.

We are now in position to recall a theorem that characterizes those GMRA's (with dilations induced by matrices of arbitrary determinant) that admit orthonormal wavelets. Given a set $S$, we denote its Lebesgue measure by $|S|$. The result that follows is known from the literature; for the details we refer to [1] and [6].

TheOREM 1.4. Suppose that $\left(V_{j}\right)$ is a GMRA such that the core space $V_{0}$ is decomposed as in Remark 1.3. Then $\left(V_{j}\right)$ admits orthonormal wavelets if and only if the following two conditions are satisfied:

(i)

$$
\left|\bigcap_{j=1}^{\infty} \Omega_{j}\right|=0
$$


(ii)

$$
\sum_{j=1}^{\infty} \sum_{i=0}^{d-1} \chi_{\Omega_{j}}\left(\xi+\beta_{i}\right)-\sum_{j=1}^{\infty} \chi_{\Omega_{j}}(B \xi)=1 \text { a.e. }
$$

If $\left(V_{j}\right)$ admits orthonormal wavelets then the dimension function of each associated orthonormal wavelet $\psi$ satisfies

$$
D_{\psi}(\xi)=\sum_{j=1}^{\infty} \chi_{\Omega_{j}}(\xi) \text { a.e. }
$$

Notice that condition (1.1) is trivially satisfied when $V_{0}$ has only finitely many, say $m$, generators, since it is understood in this situation that $\Omega_{j}=$ $\emptyset, \forall j>m$. As an immediate corollary, we state the corresponding result for PF MRA's that is most relevant for our study. Given a PF MRA $\left(V_{j}\right)$ such that $V_{0}=\langle\varphi\rangle$, the function $\varphi$ will be called a scaling function (although $\varphi$ is only a Parseval, not necessarily orthonormal generator).

Corollary 1.5. Let $\left(V_{j}\right)$ be a PF MRA with a scaling function $\varphi$ such that $\sum_{k \in \mathbb{Z}^{n}}|\hat{\varphi}(\xi+k)|^{2}=\chi_{\Omega}(\xi)$ a.e. Then $\left(V_{j}\right)$ admits orthonormal wavelets if and only if

$$
\sum_{i=0}^{d-1} \chi_{\Omega}\left(\xi+\beta_{i}\right)-\chi_{\Omega}(B \xi)=1 \text { a.e. }
$$

If $\left(V_{j}\right)$ admits orthonormal wavelets then the dimension function of each associated orthonormal wavelet $\psi$ satisfies

$$
D_{\psi}(\xi)=\chi_{\Omega}(\xi) \text { a.e. }
$$

The preceding corollary shows the difficulty that arises in the case $d=$ $|\operatorname{det} A|>2$. If $d=2$, we see that each MRA $\left(V_{j}\right)$ with an orthonormal generator for the core space $V_{0}$ admits orthonormal wavelets, since then $\Omega=$ $\mathbb{R}^{n}$ (up to a null-set) and (1.4) is obviously satisfied. In contrast, when $d>2$, if we take an orthonormal generator for $V_{0}$, the left hand side of (1.4) is equal to $d-1>1$ a.e., and, consequently, (1.4) cannot be satisfied (we note in passing that, in this situation, $\left(V_{j}\right)$ admits only orthonormal multi-wavelets with $d-1$ generators).

Thus, if we want to construct a PF MRA that admits orthonormal wavelets, we must work with Parseval generators that are not orthonormal. Roughly speaking, a construction of such PF MRA's boils down to a construction of those $\mathbb{Z}^{n}$-periodic measurable sets $\Omega$ that satisfy (1.4). In this paper we provide a method of construction of all PF MRA's $\left(V_{j}\right)$ such that the core space $V_{0}$ is of the form $V_{0}=\langle\varphi\rangle$ where $\hat{\varphi}=\chi_{S}$ and the set $\Omega=S+\mathbb{Z}^{n}$ satisfies (1.4). In this situation, we say that $S$ is a scaling set. By Corollary 1.5, such PF MRA's admit orthonormal wavelets and we shall see 
that all these wavelets belong to the MSF class. Moreover, it turns out that the relation between a scaling set $S$ and the corresponding wavelet set $W$ is essentially the same as in Proposition 1.1: $W=B S \backslash S$.

Many examples of scaling sets and wavelet sets with dyadic dilations on the real line are well known. Various methods of construction can be found in the literature (see, for example, Section 6 of [15].) Moreover, it is known from the literature that no major difficulties arise in $\mathbb{R}^{n}$ in the case $d=2$. It was shown in [12] that every expanding matrix $A \in M_{n}(\mathbb{Z})$ such that $d=|\operatorname{det} A|=2$ admits scaling sets; for this case we also refer the reader to $[3]$.

In contrast to that, construction of wavelet sets and, in particular, scaling sets in the case $d>2$ is a more difficult question, even on the real line. It is proved in [10] that every expanding matrix with integer coefficients admits wavelet sets. The first examples of wavelet sets with general dilations on the real line appeared in [9]. Further examples of wavelet sets can be found in [16], [11] and [1]; in particular [1] provides a general procedure for construction of wavelet sets. Finally, it is proved in [8] that each expanding matrix admits scaling sets and some new examples are presented. All these results and examples are obtained by techniques that differ from ours. The essence of our approach is a systematic use of the underlying multiresolution structure and, hence, the central objects of our study are scaling sets.

This concludes our description of the background of the subject. The rest of the article is organized as follows: In Section 2 we characterize all scaling sets in Theorem 2.4. In Theorem 2.8 we give a construction method of scaling sets and prove that all scaling sets arise in that way. After that, we prove in Corollary 2.10 that each expanding matrix with integer coefficients admits scaling sets. At the end of Section 2 we explain in detail how our results are related to those from [1].

Section 3 is devoted to examples. First, we analyze the series of examples on the real line presented in [9] from our viewpoint. This enables us to construct new examples in $L^{2}(\mathbb{R})$ for all dilation factors $d \in \mathbb{N}, d>2$. Finally, we present an example of a scaling set and the corresponding wavelet set in $L^{2}\left(\mathbb{R}^{2}\right)$ for a matrix $A$ such that $d=|\operatorname{det} A|=3$. The dilation matrix $A$ used in that example is similar to rotation by $\pi / 6$ composed with the dilation by $\sqrt{3}$ and may be regarded as a "generalized quincunx" matrix.

Section 4 completes our study of PF MRA's from Section 2. It is devoted to PF MRA's with general scaling functions. In Theorem 4.2 we give a description of wavelets that arise from PF MRA's in terms of the underlying multiresolution structure. In this way, we generalize the well known high pass filter technique for computing dyadic MRA wavelts. The paper ends with more examples which are constructed by using the results obtained in Section 4. 
Throughout the paper we denote by $\langle\cdot, \cdot\rangle$ the standard inner product in $\mathbb{R}^{n}$. As already indicated in our description of the Shannon wavelet and in the definition of the dimension function, we use the Fourier transform in the form $\hat{f}(\xi)=\int_{\mathbb{R}^{n}} f(x) \mathrm{e}^{-2 \pi i\langle x, \xi\rangle} d x$. For a function $\varphi \in L^{2}\left(\mathbb{R}^{n}\right)$ we denote $S_{\varphi}=\left\{\xi \in \mathbb{R}^{n}: \hat{\varphi}(\xi) \neq 0\right\}$ and $\Omega_{\varphi}=S_{\varphi}+\mathbb{Z}^{n}$. We denote by $\sigma_{\varphi}$ the periodization function of $\hat{\varphi}$ defined by $\sigma_{\varphi}(\xi)=\sum_{k \in \mathbb{Z}^{n}}|\hat{\varphi}(\xi+k)|^{2}$.

\section{SCALING SETS}

We begin with a result that characterizes those functions that are scaling functions for PF MRA's for which there is an associated orthonormal wavelet.

THEOREM 2.1. Let $\varphi \in L^{2}\left(\mathbb{R}^{n}\right)$. Then $\varphi$ is a scaling function for a PF $M R A$ that admits orthonormal wavelets if and only if the following conditions are satisfied:

(SF1) $\sigma_{\varphi}(\xi)=\chi_{\Omega_{\varphi}}(\xi)$ a.e.;

(SF2) there exists a measurable $\mathbb{Z}^{n}$-periodic function $m_{0}$ such that $\hat{\varphi}(B \xi)=$ $m_{0}(\xi) \hat{\varphi}(\xi)$ a.e. (and, hence, $B^{-1} S_{\varphi} \subseteq S_{\varphi}$, up to a set of measure zero);

(SF3) $\lim _{j \rightarrow \infty}\left|\hat{\varphi}\left(B^{-j} \xi\right)\right|=1$ a.e.;

(SF4) $h_{\varphi}(\xi):=\sum_{i=0}^{d-1} \chi_{\Omega_{\varphi}}\left(\xi+\beta_{i}\right)-\chi_{\Omega_{\varphi}}(B \xi)=1$ a.e.

Proof of Theorem 2.1. First observe that $\varphi$ is a scaling function for a PF MRA if and only if (SF1), (SF2), and (SF3) are satisfied. This is well known; we refer the reader to [13, Theorem 7.5.2] for the proof in the dyadic case on the real line. The same statement is proved in [3, Proposition 3.7] for dilations induced by expanding matrices with the property $d=2$. The general proof (for $d \geq 2$ ) can be obtained by an easy adaptation of these standard arguments; hence, we omit the details.

The role of the last condition was explained in Corollary 1.5; once we have a scaling function, (SF4) is precisely what is necessary and sufficient for the existence of associated orthonormal wavelets.

We now focus our attention on (SF4). The following lemma provides more insight into the equality $\sum_{i=0}^{d-1} \chi_{\Omega_{\varphi}}\left(\xi+\beta_{i}\right)-\chi_{\Omega_{\varphi}}(B \xi)=1$ a.e.

Lemma 2.2. Let $S \subseteq \mathbb{R}^{n}$ ba a measurable set such that $B^{-1} S \subseteq S$. Denote $\Omega=S+\mathbb{Z}^{n}$ and $h(\xi)=\sum_{i=0}^{d-1} \chi_{\Omega}\left(\xi+\beta_{i}\right)-\chi_{\Omega}(B \xi)$. Then $h(\xi)=1$ a.e. if and only if the following conditions are satisfied:

(i) $S+B^{-1} \mathbb{Z}^{n}=\mathbb{R}^{n}$, up to a set of measure zero;

(ii) for a.e. $\xi \in R:=S \backslash B^{-1} \Omega, \xi+\beta \notin S$ for all $\beta \in B^{-1} \mathbb{Z}^{n} \backslash \mathbb{Z}^{n}$;

(iii) for a.e. $\xi \in S \cap B^{-1} \Omega$, there is a unique index $j=j(\xi) \geq 1$ such that $\xi+\beta_{j(\xi)} \in \Omega$.

Proof of Lemma 2.2. Suppose that $h(\xi)=1$ a.e. First, we then have $\sum_{i=0}^{d-1} \chi_{\Omega}\left(\xi+\beta_{i}\right) \geq 1$ a.e. Thus, for all such $\xi$ 's, there exist $i \in\{0,1, \ldots, d-1\}$ 
and $k \in \mathbb{Z}^{n}$ such that $\xi+\beta_{i}+k \in S$, in other words, $\xi \in S+B^{-1} \mathbb{Z}^{n}$. This proves (i). Similarly, one gets (ii) and (iii) immediately from the assumption $h(\xi)=1$ a.e. Conversely, suppose (i), (ii) and (iii). By observing that the function $h$ is $B^{-1} \mathbb{Z}^{n}$-periodic and using (i), we see that it is enough to conclude that $h(\xi)=1$ for a.e. $\xi \in S$ and this follows immediately from (ii) and (iii).

REMARK 2.3. Suppose that, keeping the notations from the above lemma, $h(\xi)=1$ a.e. Then, in addition to (i), (ii) and (iii), we also have

(iv) $S=R \cup B^{-1} S \cup \sigma\left(B^{-1} S\right)$ where $\sigma\left(B^{-1} S\right)=\cup_{\xi \in B^{-1} S} \sigma(\xi)$ and, for $\xi \in B^{-1} S, \sigma(\xi)=\left\{\eta \in S: \eta=\xi+\beta_{j(\xi)}+k\right.$ for some $\left.k \in \mathbb{Z}^{n}\right\}$.

Observe that for $\xi \in B^{-1} S$ we have $\xi \in S \cap B^{-1} S \subseteq S \cap B^{-1} \Omega$, so, by (iii), there is a unique $j(\xi) \geq 1$ such that $\xi+\beta_{j(\xi)} \in \Omega$. Thus, there is at least one $k \in \mathbb{Z}^{n}$ with the property $\xi+\beta_{j(\xi)}+k \in S$. In general, $k$ is not unique and, consequently, $\sigma(\xi)$ can be a set of cardinality greater than 1 .

To prove (iv), we only have to show $R \cup B^{-1} S \cup \sigma\left(B^{-1} S\right) \supseteq S$. By the definition of the set $R$ (see (ii) in Lemma 2.2), this reduces to $S \cap\left(B^{-1} \Omega \backslash\right.$ $\left.B^{-1} S\right) \subseteq \sigma\left(B^{-1} S\right)$. Let us now take $\xi \in B^{-1} \Omega \cap S$. Then there exists $\eta \in S$ together with $l \in \mathbb{Z}^{n}$ and some $\beta_{k}$ such that $\xi=B^{-1} \eta+\beta_{k}+l \in S$. This implies, by the definition of $\sigma\left(B^{-1} \eta\right)$, that $\xi=B^{-1} \eta+\beta_{k}+l \in \sigma\left(B^{-1} \eta\right) \subseteq$ $\sigma\left(B^{-1} S\right)$.

We are now in position to prove a theorem that characterizes scaling sets. As on the real line, we say that a measurable set $F \subseteq R^{n}$ is a $\mathbb{Z}^{n}$-tiling domain if for a.e. $\xi \in \mathbb{R}^{n}$ there exists a unique $k \in \mathbb{Z}^{n}$ such that $\xi+k \in F$. The definition of a $B^{-1} \mathbb{Z}^{n}$-tiling domain is analogous. Clearly, a set $F$ is a $\mathbb{Z}^{n}$-tiling domain if and only if $B^{-1} F$ is a $B^{-1} \mathbb{Z}^{n}$-tiling domain.

THEOREM 2.4. A measurable set $S \subseteq \mathbb{R}^{n}$ is a scaling set if and only if the following conditions are satisfied:

(S1) $|(S+k) \cap S|=0, \forall k \in \mathbb{Z}^{n}, k \neq 0$;

(S2) $B^{-1} S \subseteq S$, up to a set of measure zero;

(S3) for a.e. $\xi \in \mathbb{R}^{n}$ there is an integer $j_{0}(\xi)$ for which $B^{-j} \xi \in S, \forall j \geq$ $j_{0}(\xi)$

(S4) $S_{0}=B^{-1} S \cup R$ is a $B^{-1} \mathbb{Z}^{n}$-tiling domain, where $R=S \backslash B^{-1} \Omega=$ $\left\{\xi \in S: \xi+\beta \notin S, \forall \beta \in B^{-1} \mathbb{Z}^{n} \backslash\{0\}\right\} ;$

(S5) for a.e. $\xi \in B^{-1} S$ there exist a unique $\beta=\beta(\xi) \in B^{-1} \mathbb{Z}^{n} \backslash\{0\}$ such that $\sigma(\xi)=\xi+\beta(\xi) \in S$. Moreover, $S=R \cup B^{-1} S \cup \sigma\left(B^{-1} S\right)$ is, up to a set of measure zero, a disjoint union.

Proof of Theorem 2.4. Let $\hat{\varphi}=\chi_{S}$. By Theorem 2.1 (see also the proof), $\varphi$ is a scaling function for a PF MRA if and only if $\varphi$ satisfies (SF1), (SF2), and (SF3). Obviously, this reduces in our situation to (S1), (S2), and (S3). 
Suppose that $S$ is a scaling set. Then, by the above observation, $S$ satisfies (S1), (S2), and (S3). Moreover, $\varphi$ is then a scaling function for a PF MRA that admits orthonormal wavelets; hence, by (SF4) from Theorem 2.1, $S$ satisfies the hypothesis of Lemma 2.2 .

Take any $\xi \in B^{-1} S$. By Lemma 2.2 (iii), there exists a unique $j(\xi) \geq 1$ such that $\xi+\beta_{j(\xi)} \in \Omega=S+\mathbb{Z}^{n}$. By (S1), ignoring a null-set, there is a unique $k \in \mathbb{Z}^{n}$ such that $\xi+\beta_{j(\xi)}+k \in S$. Let us denote $\beta(\xi)=\beta_{j(\xi)}+k$; clearly, $\beta(\xi) \in B^{-1} \mathbb{Z}^{n}$. Define $\sigma(\xi)=\xi+\beta(\xi)$. This gives us a function $\sigma: B^{-1} S \rightarrow S$.

We now claim that $\left|B^{-1} S \cap \sigma\left(B^{-1} S\right)\right|=0$.

Take any $\xi \in B^{-1} S$ and assume $\sigma(\xi) \in B^{-1} S$. This means that there is $\eta \in B^{-1} S$ such that $\xi+\beta(\xi)=\eta$ which implies $B \eta-B \xi=B(\beta(\xi)) \in \mathbb{Z}^{n}$. By (S1), ignoring a null-set, we have $B(\beta(\xi))=0$; thus $\beta(\xi)=0$. This implies $\beta_{j(\xi)}=-k \in \mathbb{Z}^{n}$ which is impossible since $j(\xi) \geq 1$.

Let us now prove that $R=S \backslash B^{-1} \Omega=\left\{\xi \in S: \xi+\beta \notin S, \forall \beta \in B^{-1} \mathbb{Z}^{n} \backslash\right.$ $\{0\}\}$. The inclusion $\subseteq$ follows from $(\mathrm{S} 1)$ and Lemma 2.2 (ii). To prove the opposite inclusion, assume that $\xi \in S, \xi+\beta \notin S, \forall \beta \in B^{-1} \mathbb{Z}^{n} \backslash\{0\}$. We must show that $\xi \notin B^{-1} \Omega$. Suppose the opposite: $\xi \in B^{-1} \Omega$. This gives us $\eta \in S$ and $k \in \mathbb{Z}^{n}$ such that $\xi=B^{-1} \eta+B^{-1} k$; thus, $\xi-B^{-1} k=B^{-1} \eta$. By (S2) and our assumption on $\xi$, this implies $k=0$, so we have $\xi=B^{-1} \eta \in B^{-1} S$. Now, by the definition of $\sigma$, we have $\sigma(\xi)=\xi+\beta(\xi) \in S$ with $\beta(\xi) \neq 0$ which contradicts the assumption on $\xi$.

Next we claim that $B^{-1} S \cup \sigma\left(B^{-1} S\right)=S \backslash R$.

To see this, first observe that $R=S \backslash B^{-1} \Omega$ implies $S \backslash R=S \cap B^{-1} \Omega$, so we must show $B^{-1} S \cup \sigma\left(B^{-1} S\right)=S \cap B^{-1} \Omega$. The inclusion $B^{-1} S \subseteq$ $S \cap B^{-1} \Omega$ is obvious, while $\sigma\left(B^{-1} S\right) \subseteq S \cap B^{-1} \Omega$ is seen in the following way: first, $\sigma\left(B^{-1} S\right) \subseteq S$ is clear and, secondly, $B(\sigma(\xi))=B \xi+B(\beta(\xi)) \in$ $\Omega, \forall \xi \in B^{-1} S$. Thus, we have proved $B^{-1} S \cup \sigma\left(B^{-1} S\right) \subseteq S \cap B^{-1} \Omega$. The opposite inclusion is already proved in Remark 2.3. Finally, we have $S=$ $R \cup B^{-1} S \cup \sigma\left(B^{-1} S\right)$ and it is clear from the preceding arguments that this union is essentially disjoint. This proves (S5).

Put $S_{0}=B^{-1} S \cup R$. Next we prove that $S_{0}$ is a $B^{-1} Z^{n}$-tiling domain.

First, by Lemma 2.2 (i), we have $S+B^{-1} Z^{n}=\mathbb{R}^{n}$, i.e., $\left(B^{-1} S \cup R \cup\right.$ $\left.\sigma\left(B^{-1} S\right)\right)+B^{-1} \mathbb{Z}^{n}=\mathbb{R}^{n}$. Notice that, by the definition of $\sigma$, we have $\sigma\left(B^{-1} S\right)+B^{-1} \mathbb{Z}^{n} \subseteq B^{-1} S+B^{-1} \mathbb{Z}^{n}$. This shows that $S_{0}+B^{-1} \mathbb{Z}^{n}=\mathbb{R}^{n}$. It remains to prove that $\left|\left(S_{0}+\beta\right) \cap S_{0}\right|=0$ for each $\beta \in B^{-1} \mathbb{Z}^{n} \backslash\{0\}$. Let us take an arbitrary $\beta \in B^{-1} \mathbb{Z}^{n} \backslash\{0\}$. First observe that $R+\beta$ does not intersect $S$; hence $(R+\beta) \cap\left(B^{-1} S \cup R\right)=\emptyset$. This gives us $\left|\left(S_{0}+\beta\right) \cap S_{0}\right|=$ $\left|\left(B^{-1} S \cup R+\beta\right) \cap\left(B^{-1} S \cup R\right)\right|=\left|\left(B^{-1} S+\beta\right) \cap\left(B^{-1} R \cup R\right)\right|$. Finally, notice that for $\xi \in B^{-1} S, \xi+\beta \in S$ implies $\beta=\beta(\xi)$; hence $\xi+\beta=\sigma(\xi)$. Thus, $\left(B^{-1} S+\beta\right) \cap\left(B^{-1} R \cup R\right) \subseteq \sigma\left(B^{-1} S\right) \cap\left(B^{-1} R \cup R\right)$ and, since the last intersection has measure zero, the argument is completed.

The converse is clear. 
REMARK 2.5. With the notations from the preceding theorem and its proof, put $F=B S_{0}$. If $S$ is a scaling set then, by (S4), $F$ is a $\mathbb{Z}^{n}$-tiling domain such that $B^{-1} F \subseteq S \subseteq F$; in particular, $B^{-1} F \subseteq F$. Moreover, since $S \subseteq F$, it is clear that $F$ satisfies (S3) as well. Recall from Proposition 1.1 , that in the dyadic case (the same applies to the case $d=2$ ), such tiling domains are scaling sets. As the preceding theorem shows, when $d>2$, scaling sets are nested between $F$ and $B^{-1} F$.

REmark 2.6. (a) Suppose that $S$ is a scaling set; put $\hat{\varphi}=\chi_{S}, V_{0}=\langle\varphi\rangle$ and $V_{j}=D^{j} V_{0}, j \in \mathbb{Z}$. If, for a subspace $V \subseteq \mathbb{R}^{n}$, we denote $\widehat{V}=\{\hat{f}: f \in V\}$, it is easy to see that $\widehat{V_{0}}=L^{2}(S), \widehat{V_{1}}=L^{2}(B S)$ and $\widehat{W_{0}}=L^{2}(B S \backslash S)$. Thus, if we denote $W=B S \backslash S, W$ is a wavelet set. It is well known that this is equivalent to the conditions $\mathbb{R}^{n}=\cup_{k \in \mathbb{Z}^{n}}(W+k)=\cup_{j \in \mathbb{Z}} B^{j} W$, up to a set of measure 0 .

(b) Since $S \subseteq B S$ we have $1=|W|=|B S \backslash S|=|B S|-|S|=d|S|-$ $|S|=(d-1)|S|$. This shows that all scaling sets have the same measure: $|S|=\frac{1}{d-1}$. From this we see again that a $\mathbb{Z}^{n}$-tiling domain can be a scaling set only when $d=2$. Also, $\left|B^{-1} S\right|=\left|\sigma\left(B^{-1} S\right)\right|=\frac{1}{d(d-1)}$ and this implies $|R|=\frac{1}{d-1}-\frac{2}{d(d-1)}=\frac{d-2}{d(d-1)}$. This shows that $|R|=0$ if and only if $S=F$ if and only if $d=2$.

Having obtained a characterization of scaling sets, we now turn to a method of construction. Our next theorem provides an algorithm for construction of scaling sets; moreover, we shall see that all scaling sets arise in that way.

First we need an auxiliary result. Suppose that $F$ is a $\mathbb{Z}^{n}$-tiling domain. Observe that $\xi \mapsto \bar{\xi}:=\xi+\mathbb{Z}^{n}$ is a $1-1$ measure-preserving correspondence between $F$ and the torus $\mathbb{T}^{n}=\mathbb{R}^{n} / \mathbb{Z}^{n}$. The group $\Gamma=B^{-1} \mathbb{Z}^{n} / \mathbb{Z}^{n}$ of order $d$ acts faithfully on $\mathbb{T}^{n}$ by translation. Since $S_{0}$ is a $B^{-1} \mathbb{Z}^{n}$-tiling domain, its image in $\mathbb{T}^{n}$ is a cross-section for the $\Gamma$-orbits. This is, essentially, the contents of the following lemma that provides us with a useful measurable partition of $F$. We denote by $\bar{B}: \mathbb{T}^{n} \rightarrow \mathbb{T}^{n}$ a group homomorphism defined by $\bar{B} \bar{\xi}=\overline{B \xi}$. Finally, for $\xi \in \mathbb{R}^{n}$, we denote by $\tau(\xi)$ the translation projection that is defined as the unique element $\tau(\xi)$ of $F$ with the property $\xi-\tau(\xi) \in \mathbb{Z}^{n}$.

LEMma 2.7. Let $F$ be a $\mathbb{Z}^{n}$-tiling domain.

(i) Let $C_{0}=\tau\left(B^{-1}\right) F$. Then $\left.\bar{B}\right|_{\overline{C_{0}}}: \overline{C_{0}} \rightarrow \bar{F}$ is a bijection.

(ii) For each $i=1, \ldots, d-1$, let $C_{i}=\tau\left(C_{0}+\beta_{i}\right)$. Then $\left\{C_{0}, C_{1}, \ldots, C_{d-1}\right\}$ is a partition of $F$.

Proof of Lemma 2.7. The proof of [3, Lemma 2.3] applies without essential changes. 
Theorem 2.8. Let $S \subseteq \mathbb{R}^{n}$. Then $S$ is a scaling set if and only if $S$ is, up to a set of measure zero, of the form $S=\cup_{j=0}^{\infty} S_{j}$ and the sequence $\left(S_{j}\right)$ is constructed by the following algorithm:

(A1) Pick a $\mathbb{Z}^{n}$-tiling domain $F$ with the properties

(i) $B^{-1} F \subseteq F$;

(ii) $S_{0}:=B^{-1} F$ satisfies (S3) from Theorem 2.4.

(A2) For $j \geq 0$ choose inductively measurable functions $\gamma_{j}: B^{-1} S_{j} \rightarrow$ $\left\{\beta_{1}, \ldots, \beta_{d-1}\right\}$ and put $\sigma_{j}(\xi)=\tau\left(\xi+\gamma_{j}(\xi)\right), \forall \xi \in B^{-1} S_{j}$. Let $S_{j+1}=\sigma_{j}\left(B^{-1} S_{j}\right)$.

Proof of Theorem 2.8. Suppose that $S=\cup_{j=0}^{\infty} S_{j}$ and that the sequence $\left(S_{j}\right)$ is constructed by the above algorithm. Since $B^{-1} F \subseteq F$, the partition of $F$ from the above lemma is here $\left\{S_{0}, \tau\left(S_{0}+\beta_{1}\right), \ldots, \tau\left(S_{0}+\beta_{d-1}\right)\right\}$. Observe that $S=\cup_{j=0}^{\infty} S_{j}$ is a disjoint union, for any choice of the sequence $\left(\gamma_{j}\right)$. Moreover, $B^{-1} S \subseteq S_{0}, S_{0}$ is a $B^{-1} \mathbb{Z}^{n}$-tiling domain, and $S_{0}=B^{-1} S \cup R$ where $R=S \backslash B^{-1} \Omega$ and $\Omega=S+\mathbb{Z}^{n}$. With the function $\sigma$ on $B^{-1} S=$ $\cup_{j=0}^{\infty} B^{-1} S_{j}$ restricting to $\sigma_{j}$ on $B^{-1} S_{j}, S$ satisfies (S1) - (S5) from Theorem 2.4 ; hence, $S$ is a scaling set.

Conversely, suppose that $S$ is a scaling set. Using the notations of Theorem 2.4, let $S_{j}=\sigma\left(B^{-1} S_{j-1}\right)$ for $j \geq 1$, and $S^{\prime}=\cup_{j=0}^{\infty} S_{j}$. By properties (S1) - (S5) from Theorem 2.4, $S^{\prime}$ is obtained from the algorithm (A1),(A2). By the first part of the proof, $S^{\prime}$ is a scaling set. Since $S^{\prime} \subseteq S$ and, by Remark 2.6, $\left|S^{\prime}\right|=|S|=\frac{1}{d-1}$, we conclude that $S \backslash S^{\prime}$ is a null-set.

REMARK 2.9. Notice that there are no conditions on the maps $\gamma_{j}$ from (A2). Each $\gamma_{j}$ simply corresponds to a partition of the set $B^{-1} S_{j}$ that consists of finitely many (up to $d-1$ ) measurable sets. When $d \geq 3$, there are uncountably many choices for $\gamma_{j}$. Hence, every choice of a $\mathbb{Z}^{n}$-tiling domain $F$ that satisfies (i) and (ii) from (A1) gives rise to an uncountable family of scaling sets $S$ and corresponding wavelet sets $W=B S \backslash S$.

As we mentioned in the introduction, the existence of scaling sets in the case $d=2$ is proved in [12]. Essentially, the argument consists of proving that, for each expanding matrix $A$ with integer coefficients, there is a $\mathbb{Z}^{n}$ tiling domain $F$ such that $B^{-1} F \subseteq F$ (up to a null-set) and that $F$ contains an open neighborhood of 0 . Observe that the later property obviously implies that $F$ satisfies (S3) from Theorem 2.4. In the case $d=2$ this was enough to conclude that $S=F$ is a scaling set (cf. Remark 2.6).

By combining this result with the preceding remark and Theorem 2.4, we conclude that scaling sets exist for all $d \geq 2$. We note that corollary that follows is not new; this result is already obtained in [8, Theorem 1] using a different argument.

COROLlary 2.10. Each expanding matrix with integer coefficients admits scaling sets. 
As we already noted in the introduction, related results known from the literature are mainly concerned with wavelet sets. In particular, a procedure for construction of all wavelet sets is obtained in [1]. Let us now show how our results are related to those from [1].

First, we need to recall the definition of a complementary pair introduced in [1]. In what follows, we denote by $C$ the unit cube in $\mathbb{R}^{n}$. Suppose that $E$ is a measurable set with the property $B^{-1} E \subseteq E$. We say that $\left(T, T^{\prime}\right)$ is a complementary pair for $E$ if $T: C \rightarrow E$ and $T^{\prime}: E \rightarrow E$ are measurable one-to-one maps that satisfy (up to a set of measure 0 )

(i) $T^{\prime}(E) \subseteq E \backslash T(C)$,

(ii) $B(T(\xi)) \equiv \xi$ and $B\left(T^{\prime}(\xi)\right) \equiv \xi\left(\right.$ where $\equiv$ denotes congruence $\left.\bmod \mathbb{Z}^{n}\right)$,

(iii) $E=\cup_{j \geq 0}\left(T^{\prime}\right)^{j}(T(C))$.

By [1, Theorem 2.2], if $E$ is a measurable set with the property $B^{-1} E \subseteq E$ and if $\left(T, T^{\prime}\right)$ is a complementary pair for $E$, then $W=B E \backslash E$ is a (subspace) wavelet set. Conversely, if $W \subseteq \mathbb{R}^{n}$ is a (subspace) wavelet set, there exists a complementary pair $\left(T, T^{\prime}\right)$ for the set $E=\cup_{j<0} B^{j} W$.

Suppose that $S$ is a scaling set. Then $W=B S \backslash S$ is a wavelet set; in particular, $W$ is a $\mathbb{Z}^{n}$-tiling domain and we can identify $W$ with the unit cube $C$. Let us define $T: W \rightarrow S$ by $T(\xi)=B^{-1} \xi$ and $T^{\prime}: S \rightarrow S$ by $T^{\prime} \xi=B^{-1} \xi$. Then both $T$ and $T^{-1}$ are injective measurable maps, $T(W)=B^{-1} W=$ $S \backslash B^{-1} S$ and $T^{\prime}(S)=B^{-1} S=S \backslash\left(S \backslash B^{-1} S\right)$. The above property (ii) is obvious and $\cup_{j \geq 0}\left(T^{\prime}\right)^{j}(T(W))=\cup_{j \geq 0} B^{-j}\left(S \backslash B^{-1} S\right)=S$. Thus, $\left(T, T^{\prime}\right)$ is a complementary pair for $S$.

In the opposite direction, we show how one can reconstruct the scaling set $S$ together with the corresponding $\mathbb{Z}^{n}$-tiling domain $F$ from a wavelet set $W$.

Let us fix a wavelet set $W$ that arises from PF MRA. Observe that the abovementioned [1, Theorem 2.2] describes wavelet sets that are associated, in general, with GMRA's. Thus, $W$ arises from a scaling set only under the additional assumption that the core space of the underlying multiresolution structure is singly generated. Denote by $\psi$ the corresponding wavelet which is given by $\hat{\psi}=\chi_{W}$. Notice that, by (1.5) in Corollary 1.5, we then have $D_{\psi}(\xi) \leq 1$ a.e.

Consider, as in [1, Theorem 2.2], a complementary pair $\left(T, T^{\prime}\right)$ for the underlying set $E=\cup_{j<0} B^{j} W$. Obviously, we have $W=B E \backslash E$. We shall show that $E$ is a scaling set for $W$.

First, it is obvious that the set $E$ satisfies

$$
B^{-1} E \subseteq E
$$


By [1, Theorem 2.1], the function $\chi_{\Omega}$, where $\Omega=E+\mathbb{Z}^{n}$, satisfies the consistency equation (1.4)

$$
\sum_{i=0}^{d-1} \chi_{\Omega}\left(\xi+\beta_{i}\right)-\chi_{\Omega}(B \xi)=1 \text { a.e. }
$$

Also by [1, Theorem 2.1], since $W$ is a wavelet set, the set $\cup_{j \in \mathbb{Z}} B^{j} E$ contains, up to a set of measure 0 , a neighborhood of the origin (this is the property that distinguishes between wavelet sets and subspace wavelet sets). This immediately implies that $E$ has the property

$$
\text { for a.e. } \xi \text { there is } j_{0} \in \mathbb{N} \text { such that } B^{-j} \xi \in E, \forall j \geq j_{0} \text {. }
$$

Since $W$ is a wavelet set, the family $\left\{B^{j} W: j \in \mathbb{Z}\right\}$ is, up to a set of measure zero, a partition of $\mathbb{R}^{n}$. Thus, we can write

$$
\begin{aligned}
D_{\psi}(\xi) & =\sum_{j=1}^{\infty} \sum_{k \in \mathbb{Z}^{n}}\left|\hat{\psi}\left(B^{j}(\xi+k)\right)\right|^{2}=\sum_{k \in \mathbb{Z}^{n}} \sum_{j=1}^{\infty} \chi_{W}\left(B^{j}(\xi+k)\right) \\
& =\sum_{k \in \mathbb{Z}^{n}} \sum_{j=1}^{\infty} \chi_{B^{-j} W}(\xi+k)=\sum_{k \in \mathbb{Z}^{n}} \chi_{\cup_{j=1}^{\infty} B^{-j} W}(\xi+k) \\
& =\sum_{k \in \mathbb{Z}^{n}} \chi_{E}(\xi+k) .
\end{aligned}
$$

This, together with our assumption $D_{\psi}(\xi) \leq 1$ a.e., immediately implies

$$
|E \cap(E+k)|=0, \forall k \in \mathbb{Z}^{n} \backslash\{0\} .
$$

From (2.1) - (2.3) and our Theorem 2.1 we see that the function $\varphi$ defined by $\hat{\varphi}=\chi_{E}$ is a scaling function for a PF MRA that admits orthonormal wavelets

Thus, the set $E$ satisfies (S1), (S2), (S3) from our Theorem 2.4 and the function $\chi_{\Omega}$ satisfies the consistency equation (1.4). Now the proof of Theorem 2.4 shows that this is enough to obtain (S4) and (S5). By Theorem 2.4, $E$ is a scaling set.

It is clear from our introductory remarks that all wavelets $\psi$ which arise from scaling sets belong to the subclass of MSF wavelets with the additional property that $D_{\psi}(\xi) \leq 1$ a.e. (cf. the last assertion of Corollary 1.5). The converse now follows immediately from the preceding discussion.

Corollary 2.11. Suppose that $\psi$ is an MSF orthonormal wavelet such that $D_{\psi}(\xi) \leq 1$ for a.e. $\xi$. Then the set $S=\cup_{j<0} B^{j} W$ is a scaling set and $W=B S \backslash S$. 


\section{EXAMPLES}

In the first part of this section we shall be concerned with examples of scaling sets and corresponding wavelet sets on the real line. Here we have, for any $d \in \mathbb{N}, d \geq 2, A=B=d$. Our standard set of representatives for the quotient group $\frac{1}{d} \mathbb{Z} / \mathbb{Z}$ will be $\left\{\beta_{0}, \ldots, \beta_{d-1}\right\}$ where $\beta_{j}=\frac{j}{d}, 0 \leq j \leq d-1$.

Let us begin with the series of examples presented in [9].

Example 3.1. $d \in \mathbb{N}, d \geq 2$. As stated in [9], Example 4.5, part 10, the set

$$
W=\left[-\frac{d}{d+1},-\frac{1}{d+1}\right) \cup\left[\frac{1}{d^{2}-1}, \frac{1}{d+1}\right) \cup\left[\frac{d}{d+1}, \frac{d^{2}}{d^{2}-1}\right)
$$

is a wavelet set.

Here we show how one can reobtain this example using our technique. Consider the set $F=\left[\frac{-d^{2}+d+1}{d^{2}-1}, \frac{d}{d^{2}-1}\right)$; clearly, $F$ is a $\mathbb{Z}$-tiling domain. If we now take $S_{0}=\frac{1}{d} F=\left[\frac{-d^{2}+d+1}{d\left(d^{2}-1\right)}, \frac{1}{d^{2}-1}\right)$ and the function $\sigma: S_{0} \rightarrow F \backslash S_{0}$ defined by

$$
\sigma(\xi)=\left\{\begin{array}{ll}
\xi-\frac{1}{d}, & \xi \in\left[\frac{1}{d(d+1)}, \frac{1}{d^{2}-1}\right) \\
\xi+\frac{1}{d}, & \text { otherwise }
\end{array},\right.
$$

the algorithm from Theorem 2.8 gives us

$$
S=\left[-\frac{1}{d+1}, \frac{1}{d^{2}-1}\right) \cup\left[\frac{1}{d+1}, \frac{d}{d^{2}-1}\right) .
$$

It is now easy to see that $W=d S \backslash S$; i.e., the set $S$ is the underlying scaling set for the orthonormal wavelet $\psi$ given by $\hat{\psi}=\chi_{W}$.

REMARK 3.2. In general, the algorithm from Theorem 2.8 enables us to construct scaling sets $S$ that are countable unions of the form $S=\cup_{j=0}^{\infty} S_{j}$ with $S_{0}=B^{-1} F$. As the preceding example shows, it is possible to have a scaling set that consists of only finitely many components. Thus, it is natural to ask if there exist connected scaling sets. It is not hard to see that the answer is negative; if $d>2$ there are no intervals in $\mathbb{R}$ that are scaling sets. In this sense, the preceding example is optimal. However, there are more scaling sets that are unions of two intervals.

ExAmPle 3.3. Let $d \geq 3$. Take any $k$ such that $1 \leq k \leq d-1$ and

$$
C=\left[\frac{k-d}{d^{2}-1}, \frac{k}{d^{2}-1}\right), \quad A=\left[\frac{d k-1}{d^{2}-1}, \frac{d k}{d^{2}-1}\right) .
$$

Then it is easy to see that $S=C \cup A$ is a scaling set. The function $\sigma$ is defined by $\sigma(\xi)=\frac{1}{d} \xi-\frac{1}{d}$ for $\xi \in A$, and $\sigma(\xi)=\frac{1}{d} \xi+\frac{k}{d}$ for $\xi \in C$.

Observe that, for $k=1$, this reduces to the scaling set $S$ from Example 3.1 . 
The corresponding wavelet set is

$$
W=d S \backslash S=\left[\frac{d(k-d)}{d^{2}-1}, \frac{k-d}{d^{2}-1}\right) \cup\left[\frac{k}{d^{2}-1}, \frac{d k-1}{d^{2}-1}\right) \cup\left[\frac{d(d k-1)}{d^{2}-1}, \frac{d^{2} k}{d^{2}-1}\right) .
$$

It can be shown that the scaling sets $S$ from the preceding example, together with the sets $-S$ complete the list of all scaling sets that consists of only two intervals.

In our next example we demonstrate a scaling set, for each $d>2$, that consists of three intervals.

EXAMPLE 3.4. Here we construct a scaling set $S$ that is a disjoint union of 3 intervals: $S=A \cup B \cup C$. The starting point will be $A$. Then $B$ will be of the form $B=\sigma\left(\frac{1}{d} A\right)$ ( $\sigma$ will be specified later), and finally $C=\sigma\left(\frac{1}{d} B\right) \cup S_{0}$ where $S_{0}=\frac{1}{d} F$ with a suitable $\mathbb{Z}$-tiling domain $F$ such that $\sigma\left(\frac{1}{d} C\right)=A$.

Before starting our construction, let us consider the measures of those three sets. Put $|A|=m$. Then $|B|=\frac{1}{d} m$ and $|C|=\frac{1}{d^{2}} m+\frac{1}{d}$. We must have $\frac{1}{d}|C|=|A|$, i.e., $\frac{1}{d}\left(\frac{1}{d^{2}} m+\frac{1}{d}\right)=m$. The only solution is $m=\frac{d}{d^{3}-1}$. Notice that then $|S|=|A|+|B|+|C|=m+\frac{1}{d} m+\frac{1}{d^{2}} m+\frac{1}{d}=\frac{1}{d-1}$ which is, by Remark 2.6 (b), precisely what we need.

Let $A=\left[a, a+\frac{d}{d^{3}-1}\right)$ with $a$ temporarily unspecified. Put $B=\frac{1}{d} A-\frac{2}{d}$. This gives $B=\left[\frac{1}{d} a-\frac{2}{d}, \frac{1}{d} a+\frac{1}{d^{3}-1}-\frac{2}{d}\right)$.

Next, take $\sigma\left(\frac{1}{d} \xi\right)=\frac{1}{d} \xi+\frac{1}{d}, \xi \in B$, and translate the left endpoint of $\sigma\left(\frac{1}{d} B\right)=\frac{1}{d} B+\frac{1}{d}$ by $\frac{1}{d}$ to the left - this extension of $\sigma\left(\frac{1}{d} B\right)$ gives us $S_{0}$. Thus, $C=\left[\frac{1}{d^{2}} a-\frac{2}{d^{2}}, \frac{1}{d^{2}} a+\frac{1}{d\left(d^{3}-1\right)}-\frac{2}{d^{2}}+\frac{1}{d}\right)$. Now we define $\sigma\left(\frac{1}{d} \xi\right)=\frac{1}{d} \xi+\frac{1}{d}, \xi \in C$. In order to ensure the equality $\sigma\left(\frac{1}{d} C\right)=\frac{1}{d} C+\frac{1}{d}=A$, it is enough to adjust one pair of endpoints, since $\sigma(C)$ and $A$ are intervals of the same measure. By considering the left endpoints we obtain the equation $\frac{1}{d^{3}} a-\frac{2}{d^{3}}+\frac{1}{d}=a$ whose only solution is $a=\frac{d^{2}-2}{d^{3}-1}$. It only remains to verify, for this choice of the value of $a$, that $F:=d S_{0}$ contains $A$ and $B$.

By the construction, we have $A=\left[\frac{d^{2}-2}{d^{3}-1}, \frac{d^{2}+d-2}{d^{3}-1}\right), B=\left[\frac{-2 d^{2}+d}{d^{3}-1}, \frac{-2 d^{2}+d+1}{d^{3}-1}\right)$, $C=\left[\frac{-2 d+1}{d^{3}-1}, \frac{(d-1)^{2}}{d^{3}-1}\right)$.

Note that $S_{0}=\left[\frac{-2 d+1}{d^{3}-1}, \frac{-2 d+1}{d^{3}-1}+\frac{1}{d}\right) \subset C$. Observe that the left endpoint of $F=d S_{0}$ coincides with the left endpoint of $B$ - this follows from the construction (and shows that the construction is tight). It is obvious that $A \subset F$. By our results, we now know that $W=d S \backslash S$ is a wavelet set.

Since $S=A \cup B \cup C$, we conclude that $d S \backslash S$ consists of 5 disjoint intervals $T_{1}, \ldots, T_{5}$, where:

$T_{1}=\left[\frac{-2 d^{3}+d^{2}}{d^{3}-1}, \frac{-2 d^{3}+d^{2}+d}{d^{3}-1}\right)$ (this is $d B$ ),

$T_{2}=\left[\frac{-2 d^{2}+d+1}{d^{3}-1}, \frac{-2 d+1}{d^{3}-1}\right)$ (this is the hole between $B$ and $C$ ),

$T_{3}=\left[\frac{(d-1)^{2}}{d^{3}-1}, \frac{d^{2}-2}{d^{3}-1}\right)$ (this is the hole between $C$ and $A$ ),

$T_{4}=\left[\frac{d^{2}+d-2}{d^{3}-1}, \frac{d(d-1)^{2}}{d^{3}-1}\right)$ (this is to the right of $A$ inside $d C$ ), 
$T_{5}=\left[\frac{d\left(d^{2}-2\right)}{d^{3}-1}, \frac{d\left(d^{2}+d-2\right)}{d^{3}-1}\right)$ (this is $\left.d A\right)$.

We end this section with an example of a scaling set in $\mathbb{R}^{2}$. Our dilation matrix will represent the operator of rotation by $\frac{\pi}{6}$ composed with dilation by $\sqrt{3}$ and, hence, it may be regarded as a generalization of the quincunx matrix.

Example 3.5. Let $A=\left[\begin{array}{rr}3 & 1 \\ -3 & 0\end{array}\right]$. Notice that $B=\left[\begin{array}{rr}3 & -3 \\ 1 & 0\end{array}\right]$ and $B^{-1}=\frac{1}{3}\left[\begin{array}{rr}0 & 3 \\ -1 & 3\end{array}\right]$. Since $B \mathbb{Z}^{2}=\{(3 x-3 y, x): x, y \in \mathbb{Z}\}=3 \mathbb{Z} \times \mathbb{Z}$, one can take $\alpha_{0}=(0,0), \alpha_{1}=(1,0), \alpha_{2}=(2,0)$; thus, $\left\{\beta_{0}=(0,0), \beta_{1}=\left(0, \frac{1}{3}\right), \beta_{2}=\right.$ $\left.\left(0, \frac{2}{3}\right)\right\}$ is a complete set of representatives for the quotient group $B^{-1} \mathbb{Z}^{2} / \mathbb{Z}^{2}$.

To apply the algorithm from Theorem 2.8 , we first choose a suitable $\mathbb{Z}^{2}$ tiling domain in $\mathbb{R}^{2}$. Let $F$ be the parallelogram bounded by the lines $y=-\frac{1}{2}$, $y=\frac{1}{2}, y=\frac{2}{3} x+\frac{1}{6}$, and $y=\frac{2}{3} x-\frac{1}{2}$. The vertices of $F$ are $F^{1}=\left(-1,-\frac{1}{2}\right)$, $F^{2}=\left(0,-\frac{1}{2}\right), F^{3}=\left(\frac{3}{2}, \frac{1}{2}\right)$, and $F^{4}=\left(\frac{1}{2}, \frac{1}{2}\right)$.

Put $S_{0}=B^{-1} F$. The vertices of $S_{0}$ are $S_{0}^{1}=\left(-\frac{1}{2},-\frac{1}{6}\right), S_{0}^{2}=\left(-\frac{1}{2},-\frac{1}{2}\right)$, $S_{0}^{3}=\left(\frac{3}{2}, \frac{1}{2}\right)$ and $S_{0}^{4}=\left(\frac{1}{2}, \frac{1}{2}\right)$. Clearly (see Figure 1$), S_{0} \subseteq F$ and $S_{0}$ contains an open neighborhood of zero; thus, $F$ and $S_{0}$ satisfy conditions (A1)(i),(ii) from Theorem 2.8.

We now proceed by applying the second part of the algorithm. Let

$$
\sigma\left(B^{-1} \xi\right)=B^{-1} \xi+\left(1, \frac{1}{3}\right), \xi \in S_{0}, \sigma\left(B^{-1} S_{0}\right)=P_{0} .
$$

The vertices of $P_{0}$ are $P_{0}^{1}=\left(\frac{5}{6}, \frac{1}{3}\right), P_{0}^{2}=\left(\frac{1}{2}, 0\right), P_{0}^{3}=\left(1, \frac{1}{6}\right)$ and $P_{0}^{4}=\left(\frac{4}{3}, \frac{1}{2}\right)$. Let

$$
\sigma\left(B^{-1} \xi\right)=B^{-1} \xi+\left(0, \frac{1}{3}\right), \xi \in P_{0}, \sigma\left(B^{-1} P_{0}\right)=Q_{0} .
$$

The vertices of $Q_{0}$ are $Q_{0}^{1}=\left(\frac{1}{3}, \frac{7}{18}\right), Q_{0}^{2}=\left(0, \frac{1}{6}\right), Q_{0}^{3}=\left(\frac{1}{6}, \frac{1}{6}\right)$ and $Q_{0}^{4}=$ $\left(\frac{1}{2}, \frac{7}{18}\right)$. Finally, let

$$
\sigma\left(B^{-1} \xi\right)=B^{-1} \xi+\left(0,-\frac{1}{3}\right), \xi \in Q_{0}, \sigma\left(B^{-1} Q_{0}\right)=S_{1} .
$$

The vertices of $S_{1}$ are $S_{1}^{1}=\left(\frac{7}{18},-\frac{1}{18}\right), S_{1}^{2}=\left(\frac{1}{6},-\frac{1}{6}\right), S_{1}^{3}=\left(\frac{1}{6},-\frac{2}{9}\right)$ and $S_{1}^{4}=\left(\frac{7}{18},-\frac{1}{9}\right)$.

We shall now iterate the preceding three steps. Let us define inductively

$$
\begin{gathered}
\sigma\left(B^{-1} \xi\right)=B^{-1} \xi+\left(1, \frac{1}{3}\right), \xi \in S_{j}, \sigma\left(B^{-1} S_{j}\right)=P_{j}, j=1,2, \ldots \\
\sigma\left(B^{-1} \xi\right)=B^{-1} \xi+\left(0, \frac{1}{3}\right), \xi \in P_{j}, \sigma\left(B^{-1} P_{j}\right)=Q_{j}, j=1,2, \ldots \\
\sigma\left(B^{-1} \xi\right)=B^{-1} \xi+\left(0,-\frac{1}{3}\right), \xi \in Q_{j}, \sigma\left(B^{-1} Q_{j}\right)=S_{j+1}, j=1,2, \ldots
\end{gathered}
$$


One may additionally observe that $B^{-6}=-\frac{1}{27} I$ where $I$ is the unit matrix. Then one obtains from (3.4), (3.5), and (3.6), for all $j \geq 0$, that

$S_{j+2}=\frac{1}{27}\left(-S_{j}+(4,-6)\right), P_{j+2}=\frac{1}{27}\left(-P_{j}+(22,2)\right), Q_{j+2}=\frac{1}{27}\left(-Q_{j}+(2,4)\right)$.

We omit the computational details.

By Theorem 2.8, it follows that

$$
S=\cup_{j=0}^{\infty}\left(S_{j} \cup P_{j} \cup Q_{j}\right)
$$

is a scaling set (see Figure 1 where $S$ is indicated as the shaded area inside $F$; the largest three parallelograms contained in $S$ are $\left.S_{0}, P_{0}, Q_{0}\right)$.

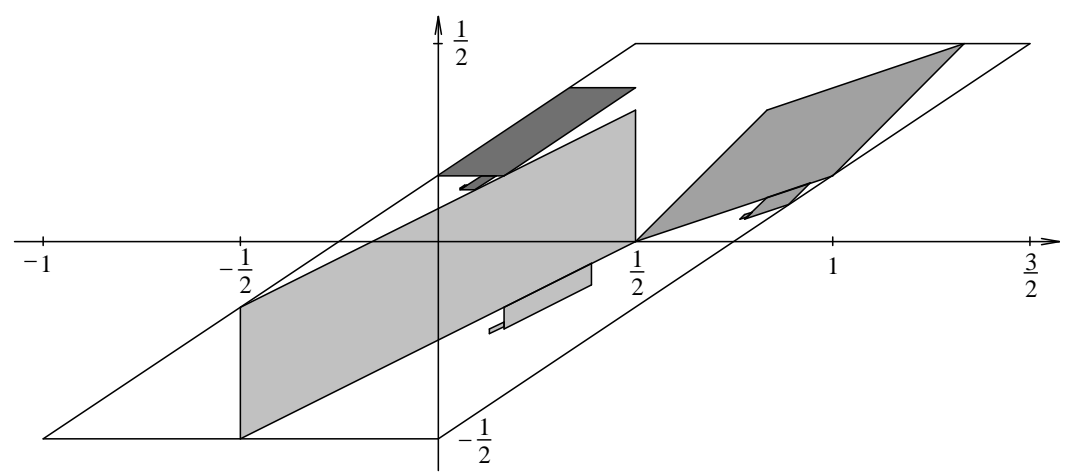

FIGURE 1

\section{PF MRA WAVELETS}

In this section we continue our study of PF MRA's. In contrast to Section 2, here we do not assume any restrictions on PF MRA's under consideration. In other words, we work with general scaling functions, not necessarily of the form $\hat{\varphi}=\chi_{S}$. The main result is Theorem 4.2 that provides a description of orthonormal wavelets that arise from PF MRA's, in terms of the underlying multiresolution structure. This will enable us to construct wavelets associated with PF MRA's with more general scaling functions. We shall make an additional comment on that in the concluding remark of the paper. In addition, we demontrate more examples that are constructed using the techniques developed in this section.

Let us begin with summarizing some standards facts concerned with the multiresolution technique. The assertions of the following remark are known 
and they will be stated without proofs. For the details, we refer the reader to the corresponding statements in $[3]^{1}$.

REMARK 4.1. Let $\left(V_{j}\right)$ be a PF MRA with a scaling function $\varphi$; put $\sigma \varphi=\chi_{\Omega}$. Then the following statements are true:

(a) For each $f \in V_{1}$ there is a unique measurable $\mathbb{Z}^{n}$-periodic function $t$ (that is called the minimal filter for $f$ ) such that $\hat{f}(B \xi)=t(\xi) \hat{\varphi}(\xi)$ a.e. and $t(\xi)=0, \forall \xi \notin \Omega$. In particular, there is a unique measurable $\mathbb{Z}^{n}$-periodic function $m_{0}$ (the minimal low-pass filter) such that $\hat{\varphi}(B \xi)=m_{0}(\xi) \hat{\varphi}(\xi)$ a.e. and $m_{0}(\xi)=0, \forall \xi \notin \Omega$. For $f \in V_{1}$ and its minimal filter $t$, we denote $t^{d}(\xi)=\left(t(\xi), t\left(\xi+\beta_{1}\right), \ldots, t\left(\xi+\beta_{d-1}\right)\right) \in$ $\mathbb{R}^{d}, \xi \in \mathbb{R}^{n}$.

(b) The minimal low-pass filter $m_{0}$ satisfies a generalized Smith-Barnwell equation: $\left\|m_{0}^{d}(\xi)\right\|=\chi_{B^{-1} \Omega}(\xi)$ a.e., where $\|\cdot\|$ denotes the Euclidean norm in $\mathbb{R}^{n}$.

(c) A function $f \in V_{1}$ belongs to $W_{0}$ if and only if its minimal filter $t$ satisfies $\left\langle t^{d}(\xi), m_{0}^{d}(\xi)\right\rangle=0$ for a.e. $\xi \in \mathbb{R}^{n}$.

(d) Suppose that $\mu$ is a measurable function which is unimodular and $\mathbb{Z}^{n}$ periodic a.e. Then $\varphi_{1}$ defined by $\hat{\varphi}_{1}(\xi)=\mu(\xi) \hat{\varphi}(\xi)$ is a scaling function for the same PF MRA $\left(V_{j}\right)$.

(e) Suppose, additionally, that $\left(V_{j}\right)$ admits orthonormal wavelets. Then a function $\psi \in W_{0}$ is an orthonormal wavelet associated with $\left(V_{j}\right)$ if and only if $\sigma_{\psi}(\xi)=1$ a.e.

[13, Proposition 2.2.13] provides a description of an MRA orthonormal wavelet with dyadic dilations on the real line in terms of the underlying MRA. The following theorem generalizes this result in two directions: first, the ambient space is $\mathbb{R}^{n}$ and, secondly, it is concerned with arbitrary dilation matrices with integer coefficients (so that $d \geq 2$ ). It provides us with an analog of the concept of a high-pass filter that appears in the aforementioned classical result. In contrast to the dyadic situation, here we obtain only an algorithm, rather than a formula, for the "high-pass filter" of the PF MRA in question. A similar result for GMRA's that admit orthonormal wavelets in the case $d=2$ is proved in [2].

ThEOREM 4.2. Let $\left(V_{j}\right)$ be a PF MRA that admits orthonormal wavelets. Then there exists a measurable $\mathbb{Z}^{n}$-periodic map $\xi \mapsto v(\xi)$ with the properties

- $\sum_{i=0}^{d-1}\left|v\left(\xi+\beta_{i}\right)\right|^{2}=1$ a.e.;

- for each $\psi \in W_{0}$ there exists a measurable $\mathbb{Z}^{n}$-periodic function s such that $\hat{\psi}(B \xi)=s(B \xi) v(\xi) \hat{\varphi}(\xi)$ a.e.

\footnotetext{
${ }^{1}$ It should be mentioned that all of these assertions in [3] are proved in $L^{2}\left(\mathbb{R}^{n}\right)$, but only in the case $d=2$. However, it is not difficult to see that all the arguments do not depend on $d$.
} 
A function $\psi \in W_{0}$ written as in 2. is an orthonormal wavelet associated with $\left(V_{j}\right)$ if and only if $s$ is unimodular a.e.

Proof of Theorem 4.2. Put $V_{0}=\langle\varphi\rangle$. By Theorem 2.1, $\sigma_{\varphi}(\xi)=$ $\chi_{\Omega}(\xi)$ a.e. for some measurable $\mathbb{Z}^{n}$-periodic set $\Omega$. Since $\left(V_{j}\right)$ admits orthonormal wavelets, we have, by Corollary 1.5,

$$
\sum_{i=0}^{d-1} \chi_{\Omega}\left(\xi+\beta_{i}\right)-\chi_{\Omega}(B \xi)=1 \text { a.e. }
$$

Let $\xi \in \mathbb{R}^{n}$.

If $\xi \notin B^{-1} \Omega$, Remark 4.1 (b) implies $m_{0}^{d}(\xi)=0$. From (4.1) we conclude that there is a unique $i, 0 \leq i \leq d-1$, such that $\xi \in \Omega-\beta_{i}$ and $\xi \notin$ $\Omega-\beta_{k}, \forall k \neq i$. By Remark 4.1 (a), we now have, for each $\psi \in W_{0}$ and the corresponding filter $t, t^{d}(\xi)=(0, \ldots, 0,1,0, \ldots, 0)$ where 1 is placed on the coordinate place $i+1$. Denote $L(\xi)=\operatorname{span}\{(0, \ldots, 0,1,0, \ldots, 0)\} \subseteq \mathbb{C}^{d}$.

If $\xi \in B^{-1} \Omega$, Remark 4.1 (b) implies $\left\|m_{0}^{d}(\xi)\right\|=1$. From (4.1) we conclude that there are precisely two indices $i$ and $j, 0 \leq i, j \leq d-1$, such that $\xi \in \Omega-\beta_{i}, \xi \in \Omega-\beta_{j}$ and $\xi \notin \Omega-\beta_{k}, \forall k \neq i, j$. Thus, by Remark 4.1 (a), (c), for any $\psi \in W_{0}$ and the corresponding filter $t, t^{d}(\xi)$ must be contained in a 1-dimensional subspace $L(\xi)$ of $\mathbb{C}^{d}$ (since both $m_{0}^{d}(\xi)$ and $t^{d}(\xi)$ must have $d-2$ trivial coordinates and non-trivial coordinates are possible only at coordinate places $i+1$ and $j+1, L(\xi)$ is in fact the orthogonal complement of a 1-dimensional subspace of $\mathbb{C}^{2}$ ).

Note that in the preceding two paragraphs we have ignored a null-set that consists of those point $\xi$ for which the equalities from Remark 4.1 (b), (c) and (4.1) are not satisfied. From the preceding discussion we conclude: for a.e. $\xi \in \mathbb{R}^{n}$ there exists a 1-dimensional subspace $L(\xi)$ of $\mathbb{C}^{d}$ such that $t^{d}(\xi) \in L(\xi)$, for all $\psi \in W_{0}$. We shall now fix a unit vector (and, hence, a basis) in $L(\xi)$, for each such $\xi$. It suffices to do that for all $\xi \in F$, where $F$ is any $\mathbb{Z}^{n}$-tiling domain, since all our functions are $\mathbb{Z}^{n}$-periodic.

Consider the partition $\left\{C_{0}, C_{1}, \ldots, C_{d-1}\right\}$ of $F$ from Lemma 2.7. First, let us choose a unit vector $v^{d}(\xi)=\left(v_{0}(\xi), v_{1}(\xi), \ldots, v_{d-1}(\xi)\right) \in L(\xi)$, for each $\xi \in C_{0}$. By the preceding conclusions, for each $\psi \in W_{0}$ and the corresponding filter $t$, there is a scalar valued function $\xi \mapsto \lambda(\xi), \xi \in C_{0}$, such that

$$
t^{d}(\xi)=\lambda(\xi) v^{d}(\xi), \xi \in C_{0} .
$$

Extend $\lambda(\xi)$ by $\mathbb{Z}^{n}$-periodicity.

Now, let $\xi \in C_{i}, 1 \leq i \leq d-1$. Recall from Lemma 2.7 that $\xi \mapsto \tau\left(\xi+\beta_{i}\right)$ is a bijection from $C_{0}$ onto $C_{i}$. Thus, $\xi-\beta_{1} \in C_{0}$, up to an integer translation, so that $v^{d}\left(\xi-\beta_{i}\right)=\left(v_{0}\left(\xi-\beta_{i}\right), v_{1}\left(\xi-\beta_{i}\right), \ldots, v_{d-1}\left(\xi-\beta_{d-1}\right)\right)$ is already defined. By (4.2), we have

$\left(t\left(\xi-\beta_{i}\right), \ldots, t\left(\xi+\beta_{d-1}-\beta_{i}\right)\right)=\lambda\left(\xi-\beta_{i}\right)\left(v_{0}\left(\xi-\beta_{i}\right), \ldots, v_{d-1}\left(\xi+\beta_{d-1}-\beta_{i}\right)\right)$. 
In particular, this shows that $t(\xi)=\lambda\left(\xi-\beta_{i}\right) v_{i}\left(\xi-\beta_{i}\right)$ or, equivalently,

$$
t\left(\xi+\beta_{i}\right)=\lambda(\xi) v_{i}(\xi), \xi \in C_{0} .
$$

Let us now define a $\mathbb{Z}^{n}$-periodic function $v$ on $F$ using the partition of $F$ from the preceding lemma. Let $v(\xi)=v_{0}(\xi), \forall \xi \in C_{0}$, and $v\left(\tau\left(\xi+\beta_{i}\right)\right)=$ $v_{i}(\xi), \forall \xi \in C_{0}, \forall i=1,2, \ldots, d-1$.

We also extend $\lambda$ from $C_{0}$ to $F=\cup_{i=0}^{d-1} C_{i}$ by $\lambda\left(\tau\left(\xi+\beta_{i}\right)\right)=\lambda(\xi), \xi \in$ $C_{0}, i \geq 1$. Now (4.3) can be rewritten in the form

$$
t(\xi)=\lambda(\xi) v(\xi) \text {, for a.e. } \xi \in F \text {. }
$$

Finally, let $s(B \xi)=\lambda(\xi)$. Clearly, $s$ is a measurable, $\mathbb{Z}^{n}$-periodic and bounded function, and (4.4) now becomes $t(\xi)=s(B \xi) v(\xi)$. Since all the preceding conclusions are obtained for an arbitrary $\psi \in W_{0}$, we have the desired equality $\hat{\psi}(B \xi)=s(B \xi) v(\xi) \hat{\varphi}(\xi)$ a.e., for all $\psi \in W_{0}$. Moreover, we have $\sum_{i=0}^{d-1}\left|v\left(\xi+\beta_{i}\right)\right|^{2}=1$ a.e. By a standard computation, it follows that $\sigma_{\psi}(B \xi)=|s(B \xi)|^{2} \sum_{i=0}^{d-1}\left|v\left(\xi+\beta_{i}\right)\right|^{2}=|s(B \xi)|^{2}$ a.e. Using Remark 4.1 (e), we conclude that $\psi$ is an orthonormal wavelet if and only if the function $s$ is unimodular a.e.

The preceding proposition together with its proof provides us with an alternative argument to prove the relation between a scaling set $S$ and the corresponding wavelet set $W$ stated in Remark 2.6 (a): $W=B S \backslash S$. The following remark should also be compared with the proof of Theorem 2.4.

REMARK 4.3. Suppose that $S$ is a scaling set. Then, by definition, $\hat{\varphi}=\chi_{S}$ is a scaling function for a PF MRA $\left(V_{j}\right)$ that admits orthonormal wavelets. In particular, If we denote $\Omega=S+\mathbb{Z}$, the set $\Omega$ satisfies equality (4.1).

In this situation the low-pass filter $m_{0}$ is given by $m_{0}=\chi_{B^{-1} S+\mathbb{Z}^{n}}$. By the proof of Theorem 2.4 and Remark 2.5, there is a $\mathbb{Z}^{n}$-tiling domain $F$ such that $B^{-1} S \subseteq B^{-1} F \subseteq S \subseteq F$. Recall from Theorem 2.4 that $F=B R \cup S$ and observe that this implies $C_{0}=B^{-1} F=R \cup B^{-1} S$, where $C_{0}$ is the set from Lemma 2.7.

From the preceding theorem we know that each orthonormal wavelet $\psi$ associated with $\left(V_{j}\right)$ is of the form $\hat{\psi}(B \xi)=s(B \xi) v(\xi) \hat{\varphi}(\xi)$. Obviously, to obtain all these wavelets, it is enough to compute the function $v$ - the simplest wavelet associated with $\left(V_{j}\right)$ will be then obtained by taking $s \equiv 1$.

In order to obtain $v$ it is enough to compute $v^{d}(\xi)=\left(v_{0}(\xi), v_{1}(\xi), \ldots\right.$, $\left.v_{d-1}(\xi)\right)$ for $\xi \in C_{0}=B^{-1} F=R \cup B^{-1} S$.

By an easy computation (we omit the details) one finds:

If $\xi \in R$, then $v_{0}(\xi)=1$ and $v_{j}(\xi)=0, \forall j \geq 1$. In particular, $v(\tau(\xi+$ $\left.\left.\beta_{j}\right)\right)=0, \forall \xi \in R, \forall j \geq 1$.

If $\xi \in B^{-1} S$ and if $i>0$ is the unique index greater than zero with the property $\xi+\beta_{i} \in \Omega$ (see Theorem 2.4), then $v_{i}(\xi)=1$ and $v_{j}(\xi)=0, \forall j \neq i$. Hence, $v\left(\tau\left(\xi+\beta_{i}\right)\right)=v_{i}(\xi)=1$. Observe that, by the definition of the map 
$\sigma$, we have $\tau\left(\xi+\beta_{i}\right)=\sigma(\xi)$. Thus, $v(\sigma(\xi))=1, \forall \xi \in B^{-1} S$. In addition, we have $v\left(\tau\left(\xi+\beta_{j}\right)\right)=0, \forall \xi \in B^{-1} S, \forall j \neq i$.

This shows that $v=\chi_{R \cup \sigma\left(B^{-1} S\right)}$ on $F$. It only remains to extend $v$ by $\mathbb{Z}^{n}$-periodicity. By Theorem 4.2 , we now have $\hat{\psi}(B \xi)=v(\xi) \hat{\varphi}(\xi)=$ $\chi_{R \cup \sigma\left(B^{-1} S\right)}(\xi) \chi_{S}(\xi)=\chi_{R \cup \sigma\left(B^{-1} S\right)}(\xi)$; in other words, $\hat{\psi}=\chi_{B R \cup B \sigma\left(B^{-1} S\right)}=$ $\chi_{B S \backslash S}$.

REMARK 4.4. We are now in position to reobtain the assertion of Corollary 2.11. Here we include the proof based on Theorem 4.2 as an illustration of the technique developed in this section.

Let $\psi$ be an orthonormal MSF wavelet such that $D_{\psi}(\xi) \leq 1$ a.e. We shall show that there exists a scaling set $S$ such that $\hat{\psi}=\chi_{B S \backslash S}$.

Since $\psi$ is an MSF orthonormal wavelet, there exists a set $W$ such that $\hat{\psi}=\chi_{W}$. We have already observed that $\psi$ arises from a PF MRA $\left(V_{j}\right)$ with a scaling function $\varphi$. The proof consists of finding a new scaling function $\varphi_{1}$ for $\left(V_{j}\right)$ that is of the form $\varphi_{1}=\chi_{S}$.

Claim: Let $S=\{\xi: \hat{\varphi}(\xi) \neq 0\}$. Then $|\hat{\varphi}(\xi)|=1$ for a.e. $\xi \in S$.

Let us first show that the desired conclusion follows easily from the above claim. Define a new function $\mu$ by $\mu(\xi)=\left\{\begin{array}{cc}\frac{1}{\hat{\varphi}(\xi)}, & \xi \in S, \\ 1, & \xi \notin S .\end{array}\right.$ Clearly, $\mu$ is a measurable function. By our claim, $\mu$ is unimodular and, since $\sum_{k \in \mathbb{Z}^{n}} \mid \hat{\varphi}(\xi+$ $k) \mid=\chi_{\Omega}(\xi)$ a.e., $\mathbb{Z}^{n}$-periodic. Hence, by Remark $4.1(\mathrm{~d}), \hat{\varphi_{1}}=\mu \hat{\varphi}=\chi_{S}$ is a new scaling function for the same PF MRA $\left(V_{j}\right)$.

It remains to prove the claim.

Take $\xi \in S$. Since $W$ is a wavelet set, we have $\mathbb{R}^{n}=\cup_{j \in \mathbb{Z}} B^{j} W$, up to a set of measure zero. Thus, by ignoring this set, we can find $m \in \mathbb{Z}$ such that $\xi \in B^{m} W$. This gives us $1=\chi_{W}\left(B^{m} \xi\right)=\hat{\psi}\left(B^{m} \xi\right)=$ $s\left(B^{m} \xi\right) v\left(B^{m-1} \xi\right) \hat{\varphi}\left(B^{m-1} \xi\right)$ wherer $s$ and $v$ are the functions from Theorem 4.2. Since $|s(\xi)|=1, \hat{\varphi}(\xi) \mid \leq 1$, and $\left|m_{0}(\xi)\right| \leq 1$ for a.e. $\xi$ (see Remark 4.1 and the proof of Theorem 4.2), this implies $\left|v\left(B^{m-1} \xi\right)\right|=1$ and $\left|\hat{\varphi}\left(B^{m-1} \xi\right)\right|=1$. Now the equality $\hat{\varphi}(B \xi)=m_{0}(\xi) \hat{\varphi}(\xi)$ shows that $\left|\hat{\varphi}\left(B^{j} \xi\right)\right|=1, \forall j \leq m-1$. Moreover, since $\left(v\left(B^{m-1} \xi\right), v\left(B^{m-1} \xi+\beta_{1}\right), \ldots, v\left(B^{m-1} \xi+\beta_{d-1}\right)\right)$ is a unit vector and $\left|v\left(B^{m-1} \xi\right)\right|=1$, we have $v\left(B^{m-1} \xi+\beta_{1}\right)=0, \forall i=1, \ldots, d-1$. Now $\left(v\left(B^{m-1} \xi\right), \ldots, v\left(B^{m-1} \xi+\beta_{d-1}\right)\right) \perp\left(m_{0}\left(B^{m-1} \xi\right), \ldots, m_{0}\left(B^{m-1} \xi+\beta_{d-1}\right)\right)$ implies $m_{0}\left(B^{m-1} \xi\right)=0$; hence $\hat{\varphi}\left(B^{m} \xi\right)=0$. By induction, we conclude that $\hat{\varphi}\left(B^{j} \xi\right)=0, \forall j \geq m$. Thus, since $\xi \in S$, we must have $m>0$ and, consequently, $|\hat{\varphi}(\xi)|=1$.

This completes our description of scaling sets and the corresponding MSF wavelets. We end the paper with another two series of examples that are obtained using the technique from the proof of Theorem 4.2. Before presenting our examples, we shall state a general remark in order to explain our construction scheme. 
REMARK 4.5. For simplicity, we restrict ourselves to the real line. Let us fix $d \in \mathbb{N}, d>2$. Let $A=B=d$ and $\beta_{i}=\frac{i}{d}, i=0,1, \ldots, d-1$.

Denote by $F$ the unit interval: $F=\left[-\frac{1}{2}, \frac{1}{2}\right)$. Let $S_{0}=\frac{1}{d} F=\left[-\frac{1}{2 d}, \frac{1}{2 d}\right)$. We wish to construct a scaling set $S$ that will satisfy $S_{0} \subseteq S \subseteq F$. Observe that this immediately implies (S1), (S2), and (S3) from Theorem 2.8. Let us denote $\Omega=S+\mathbb{Z}$ and $h(\xi)=\sum_{i=0}^{d-1} \chi_{\Omega}\left(\xi+\beta_{i}\right)-\chi_{\Omega}(d \xi)$. In view of Theorem 2.1 , it only remains to achieve $h(\xi)=1$ a.e. Observe that $\chi_{\Omega}$ is a $\mathbb{Z}$-periodic function; hence, the function $h$ is $\frac{1}{d}$-periodic. This means that it will be enough to ensure $h(\xi)=1$ for a.e. $\xi$ from a $\frac{1}{d} \mathbb{Z}$-tiling domain (typically, this will be an interval, or a finite union of intervals). The idea of our construction is to find a function $\chi_{\Omega}$ (i.e., a set $\Omega$ ) with this property. In the examples below this will be done by using the following scheme: (For simplicity we shall write $D$ instead of $\chi_{\Omega}$. Notice that this is in accordance with the last assertion of Corollary 1.5; what we really do is a construction of the dimension function of an orthonormal wavelet.)

(D1) Fix a $\frac{1}{d} \mathbb{Z}$-tiling domain $T \subseteq F$ disjoint from $S_{0}$. Put $N=F \backslash\left(S_{0} \cup T\right)$. Observe that $F=S_{0} \cup N \cup T$ is a disjoint union.

(D2) Put $D(\xi)=\left\{\begin{array}{ll}1, & \xi \in S_{0} \\ 0, & \xi \in N\end{array}\right.$. Extend $D$ to $\left(S_{0} \cup N\right)+\mathbb{Z}$ by $\mathbb{Z}$-periodicity.

(D3) Let $h(\xi)=\sum_{i=0}^{d-1} D\left(\xi+\beta_{i}\right)-D(d \xi)$. Define $D$ on $T$ with values in $\{0,1\}$ in such a way that $h(\xi)=1$ for a.e. $\xi \in T$. Extend $D$ to $T+\mathbb{Z}$ by $\mathbb{Z}$-periodicity.

(D4) Put $S=\{\xi \in F: D(\xi)=1\}$. Then $S$ is a scaling set and, consequently, $W=d S \backslash S$ is a wavelet set.

EXAMPLE 4.6. $d=2 n+1, n \in \mathbb{N}$. With the notations from the preceding remark, we have $S_{0}=\left[-\frac{1}{2(2 n+1)}, \frac{1}{2(2 n+1)}\right)$. Let $T=\left[-\frac{1}{2},-\frac{1}{2}+\frac{1}{2 n+1}\right)=$ $\left[-\frac{1}{2}, \frac{-2 n+1}{2(2 n+1)}\right)$ and $N=F \backslash\left(S_{0} \cup T\right)$. As in (D2), let $D(\xi)=1, \xi \in S_{0}$, and $D(\xi)=0, \xi \in N$; in addition, we extend $D$ to $\left(S_{0} \cup N\right)+\mathbb{Z}$ by $\mathbb{Z}$-periodicity. The key idea is now to define $D$ on $T$ using the equality

$$
D(\xi)=-\sum_{i=1}^{2 n} D\left(\xi+\beta_{i}\right)+D((2 n+1) \xi)+1, \xi \in T .
$$

Obviously, this will ensure (D3). To do that, consider the intervals

$$
\begin{gathered}
L_{k}=\left[-\frac{1}{2}+\frac{1}{(2 n+1)^{k+1}},-\frac{1}{2}+\frac{n}{(2 n+1)^{k+1}}\right), k=0,1,2, \ldots, \\
J_{k}=\left[-\frac{1}{2}+\frac{n}{(2 n+1)^{k+1}},-\frac{1}{2}+\frac{n+1}{(2 n+1)^{k+1}}\right), k=0,1,2, \ldots, \\
I_{k}=\left[-\frac{1}{2}+\frac{n+1}{(2 n+1)^{k+1}},-\frac{1}{2}+\frac{1}{(2 n+1)^{k}}\right), k=0,1,2, \ldots
\end{gathered}
$$


One can easily verify that

$$
\begin{gathered}
T=\cup_{k=1}^{\infty}\left(L_{k} \cup J_{k} \cup I_{k}\right), \\
J_{0}=S_{0}, L_{0} \cup I_{0}=N, \\
(2 n+1) L_{k}=L_{k-1}-n, \forall k>0, \\
(2 n+1) J_{k}=J_{k-1}-n, \forall k>0, \\
(2 n+1) I_{k}=I_{k-1}-n, \forall k>0 .
\end{gathered}
$$

It is now clear that these equalities enable us to use (4.5) inductively to define the function $D$ on $T$ (and, a posteriori, on $T+\mathbb{Z}$ ). It turns out that $D(\xi)=1, \forall \xi \in J_{k}$, and $D(\xi)=0, \forall \xi \in L_{k} \cup I_{k}, \forall k \in \mathbb{N}$. Thus, when we take into account that $D(\xi)=1, \forall \xi \in S_{0}=J_{0}$, we conclude, by Remark 4.5, that

$$
S=\{\xi \in F: D(\xi)=1\}=\cup_{k=0}^{\infty} J_{k}
$$

is a scaling set.

EXAmple 4.7. Let $d=2 n, n \in N$. The construction is essentially the same as the preceding one. The only difference is that the unit interval $F$ contains an even number of subintervals of length $\frac{1}{d}$, so that our set $T$ is going to be a union of two intervals.

First note that $S_{0}=\left[-\frac{1}{4 n}, \frac{1}{4 n}\right)$. Let $T=T^{-} \cup T^{+}$where $T^{-}=\left[-\frac{1}{2},-\frac{1}{2}+\right.$ $\left.\frac{1}{4 n}\right)$ and $T^{+}=-T^{-}=\left[\frac{1}{2}-\frac{1}{4 n}, \frac{1}{4 n}\right)$. As before, let $N=F \backslash\left(S_{0} \cup T\right)$ and $D(\xi)=1, \xi \in S_{0}, D(\xi)=0, \xi \in N$. Again, we extend $D$ to $\left(S_{0} \cup N\right)+\mathbb{Z}$ by $\mathbb{Z}$-periodicity. As in preceding example we will define $D$ on $T$ by

$$
D(\xi)=-\sum_{i=1}^{2 n-1} D\left(\xi+\beta_{i}\right)+D(2 n \xi)+1, \xi \in T .
$$

To do that, we need a partition of $T$ similar to the preceding one. Let

$$
a_{k}=\frac{1}{2(2 n+1)}\left(1-\frac{1}{2^{2 k} n^{2 k}}\right), k=0,1, \ldots
$$

and

$$
\begin{gathered}
A_{k}=\left[-\frac{1}{2}+a_{k-1},-\frac{1}{2}+a_{k-1}+\frac{1}{2} \frac{1}{2^{2 k} n^{2 k}}\right), k=1,2, \ldots, \\
B_{k}=\left[-\frac{1}{2}+a_{k-1}+\frac{1}{2} \frac{1}{2^{2 k} n^{2 k}},-\frac{1}{2}+a_{k}\right), k=1,2, \ldots, \\
C_{k}=\left[-\frac{1}{2}+a_{k+1}+\frac{1}{2} \frac{1}{2^{2 k+2} n^{2 k+2}},-\frac{1}{2}+a_{k}+\frac{1}{2} \frac{2 n-1}{2^{2 k+1} n^{2 k+1}}\right), k=1,2, \ldots, \\
D_{k}=\left[-\frac{1}{2}+a_{k}+\frac{1}{2} \frac{2 n-1}{2^{2 k+1} n^{2 k+1}},-\frac{1}{2}+a_{k}+\frac{1}{2} \frac{1}{2^{2 k} n^{2 k}}\right), k=1,2, \ldots
\end{gathered}
$$

Next, we note the following relations; a verification is left to the reader.

$$
T^{-}=\cup_{k=1}^{\infty}\left(A_{k} \cup B_{k} \cup C_{k} \cup D_{k}\right),
$$




$$
\begin{gathered}
2 n A_{1}+n=\left[0, \frac{1}{4 n}\right), 2 n B_{1}+n=\left[\frac{1}{4}, \frac{1}{2}-\frac{1}{4 n}\right), \\
2 n A_{k}+n=-D_{k-1}, \quad \forall k>1, \\
2 n B_{k}+n=-C_{k-1}, \quad \forall k>1, \\
2 n C_{k}+n=-B_{k}, \quad \forall k \geq 1, \\
2 n D_{k}+n=-A_{k}, \quad \forall k \geq 1 .
\end{gathered}
$$

In addition, similar relation hold for the sets $-A_{k},-B_{k},-C_{k}$, and $-D_{k}$, $k \geq 1$, which make up a partition of $T^{+}=-T^{-}$. These relations enable us to define $D$ on $T$ using (4.11). First, using (4.13), one defines $T$ on $A_{1}$ and $B_{1}$ and, by symmetry, on $-A_{1}$ and $-B_{1}$. After that, we use (4.16) and (4.17) to define $D$ on $C_{1}, D_{1},-C_{1}$, and $-D_{1}$. Proceed by induction. It turns out that $D$ maps $F$ onto $\{0,1\}$ and

$$
S=\{\xi \in F: D(\xi)=1\}=S_{0} \cup \cup_{k=1}^{\infty}\left(A_{k} \cup D_{k} \cup\left(-A_{k}\right) \cup\left(-D_{k}\right)\right) .
$$

By Remark 4.5, $S$ is a scaling set.

Concluding Remark. The results of the paper provide a complete description as well as a method of construction of all scaling sets. All wavelets that arise from our construction method are MSF-wavelets; thus, a posteriori, one obtains all wavelet sets that arise from scaling sets. It should be noted that [1] provides a method for constructing all wavelet sets (i.e., not only those that arise from PF MRA's). However, we believe that our analysis of scaling sets provides some new insight into the subject and, as demonstrated through a series of examples, can be efficiently used as a construction method.

Moreover, there is some evidence that our approach can be extended to a method of construction of non-MSF wavelets. The first step along that line is the following observation: if $\varphi_{1}$ is a scaling function of a PF MRA wavelet, then it is relatively easy to prove that there exists a scaling set $S$ contained in the set $\operatorname{supp} \hat{\psi_{1}}$. In the opposite direction, one should start with a scaling set $S$ and try to change the values of the function $\chi_{S}$ on a suitable subset of $S+\mathbb{Z}^{n}$ in order to obtain a new scaling function $\varphi_{1}$ such that the support $S_{1}$ of $\hat{\varphi}_{1}$ contains $S$ and satisfies $B^{-1} S_{1} \subset S_{1}$. Such a construction, followed by an application of Theorem 4.2, will result with a non-MSF wavelet(s).

More details, together with first examples of non-MSF wavelets with general dilations, will appear elsewhere.

\section{REFERENCES}

[1] L. Baggett, H. Medina and K. Merrill, Generalized multiresolution analyses, and a construction procedure for all wavelet sets in $\mathbb{R}^{n}$, J. Fourier Anal. Appl. 5 (1999), 563-573. 
[2] D. Bakić, Semi-orthogonal Parseval frame wavelets and generalized multiresolution analyses, Appl. Comput. Harmon. Anal. 21 (2006), 281-304.

[3] D. Bakić, I. Krishtal and E .N. Wilson, Parseval frame wavelets with $E_{n}^{(2)}$-dilations, Appl. Comput. Harmon. Anal. 19 (2005), 386-431.

[4] J. J. Benedetto and $\mathrm{S}$. Li, The theory of multiresolution analysis frames and applications to filter banks Appl. Comput. Harmon. Anal. 5 (1998), 389-427.

[5] M. Bownik, The structure of shift-invariant subspaces of $L^{2}\left(\mathbb{R}^{n}\right)$, J. Funct. Anal. 112 (2000), 282-309.

[6] M. Bownik and Z. Rzeszotnik, On the existence of multiresolution analysis for framelets, preprint, 2004.

[7] M. Bownik, Z. Rzeszotnik and D. Speegle, A characterization of dimension function of orthonormal wavelets, Appl. Comput. Harmon. Anal. 10 (2001), 71-92.

[8] X. Dai, Y. Diao, Q. Gu and D. Han, Wavelets with frame multiresolution analysis, J. Fourier Anal. Appl. 9 (2003), 39-48.

[9] X. Dai and D. Larson, Wandering vectors for unitary systems and orthogonal wavelets, Mem. Amer. Math. Soc. 134 (1998).

[10] X. Dai, D. Larson and D. Speegle, Wavelet sets in $\mathbb{R}^{n}$, J. Fourier Anal. Appl. 3 (1997), $451-456$.

[11] X. Dai, D. Larson and D. Speegle, Wavelet sets in $\mathbb{R}^{n}, I I$, Contemp. Math. 216 (1998), $15-40$.

[12] Q. Gu and D. Han, On multiresolution analysis wavelets in $\mathbb{R}^{n}$, J. Fourier Anal. Appl. 6 (2000), 437-447.

[13] E. Hernández and G. Weiss, A first course on wavelets, CRC Press, Boca Raton, 1996.

[14] M. Papadakis, On the dimension function of orthonormal wavelets, Proc. Amer. Math. Soc. 128 (2000), 2043-2049.

[15] M. Papadakis, H. Šikić and G. Weiss, The characterization of low pass filters and some basic properties of wavelets, scaling functions and related concepts, J. Fourier Anal. Appl. 5 (1999), 496-521.

[16] P. Soardi and D. Weiland, Single wavelets in $n$ dimensions, J. Fourier Anal. Appl. 4 (1998), 299-315.

D. Bakić

Department of Mathematics

University of Zagreb

Zagreb

Croatia

E-mail: bakic@math.hr

E. N. Wilson

Washington University in St. Louis

St. Louis

USA

Received: 22.12.2009.

Revised: 18.3.2010. 